\title{
Halitosis Treatment Through the Administration of Antibiotic-Resistant Probiotic Lactic Yeast Kluyveromyces marxianus fragilis B0399 (K-B0399)
}

\author{
Cecchini $\mathrm{F}^{* 1}$, Nobili $\mathrm{A}^{2}$, Zanvit $\mathrm{A}^{3}$, Miclavez $\mathrm{A}^{4}$ and Nobili $\mathrm{P}^{3,5}$ \\ ${ }^{1}$ Head Office and Research Department of Scientific and Technological Park, Italy \\ ${ }^{2}$ Nobili Dental Office, Italy \\ ${ }^{3}$ Biological Dentistry Department, Istituto Stomatologico Italiano, Italy \\ ${ }^{4}$ Miclavez Dental Office, Italy \\ ${ }^{5}$ Department of Surgical Sciences and Integrated Diagnostics, University of Genova, Italy
}

Received: 監:November 23 2018; Published: 㭗: December 11, 2018

*Corresponding author: Cecchini F, Turval Laboratories Ltd, Head Office and Research Department Scientific and Technological Park, Italy

\begin{abstract}
Halitosis (oral malodour) is a common problem, which creates severe social and relational difficulties. The most popular approach is to mask the smell, without considering the bacterial aetiology of the problem. The aim of this study is to demonstrate the efficacy of the administration of encapsulated Kluyveromyces marxianus B0399 by measuring the amount of VSC (Volatile Sulphur Compounds) with follow-up at 7 and 14 days. At the end of treatment, about $91 \%$ of patients did not present halitosis. The protocol did not consider any oral hygiene treatment. The drug is in capsule form with a shell. Therefore, results were achieved with a direct action on the intestine, not at an oral level. In conclusion, this study proves that, besides the rebalancing effect of probiotics in dysbiosis, there are many immunological implications on GALT (Gut Associated Lymphoid Tissue).

Keywords: Halitosis; Kluyveromyces Marxianus B0399; Probiotic; Probiotic Lactic Yeast; Biosympa ${ }^{\circledR}$
\end{abstract}

Abbreviations: VSC: Volatile Sulphur Compounds; GALT: Gut Associated Lymphoid Tissue; WHO: World Health Organization; SCFAs: Short-Chain Fatty Acids

\section{Introduction}

Halitosis is an organic disorder that manifests as unpleasant smell that comes from the mouth during respiration or during simple eloquence. Causes may result from imbalance of the oral cavity microbiota such as metabolic disorders, disturbed commensal microflora equilibrium, use of certain types of foods and some respiratory infections [1,2]. Everyone in life has suffered from halitosis, so much so that it has been discussed since Greek and Roman times. More detailed studies have only been done since the beginning of the last century. Miyazaki et al. [3] classified halitosis in three categories: physiological or transient bad breath, true halitosis and halitophobia or imaginary halitosis. Physiological halitosis is a situation normally present in specific situations and that goes away by itself, thus without a pathological significance. The so-called "morning breath" is a condition that is frequently presented in almost everyone. This is because during the night, the salivary flow is greatly reduced which leaves the mouth dry and smelly.

The same condition is found in people who speak a lot for work (teachers, informants, people involved in public relations, etc.). It can arise in a transient halitosis state in women during their menstrual cycle and in those who fast for long periods of time. In up to $90 \%$ of cases, real halitosis is instead a pathological condition that is a result of gastrointestinal or oro-laryngeal problems [4]. Halitosis can also be caused by some pharmacological therapies or bad habits like alcohol abuse and cigarette smoking, but also spicy food or onions, garlic and leek. Another predisposed category includes who has metabolic diseases like diabetes, hepatitis, kidney failure. Halitophobia is, lastly, a condition where the patient 
believes he or she has bad breath while not objectively experiencing that state. These types of patients need psychological support. The origin of the offensive smell derives from the degradation of peptides containing sulphur by Gram-negative anaerobes which produces the so-called VSC (Volatile Sulphur Compounds).

The most represented are hydrogen sulphate, methyl mercaptan, dimethyl sulphide, fatty and short-chain acids (butyric acid, valeric, isovaleric and propionic) compounds diamminic, poliamminic, indole (skatole, methylamine, putrescine, cadaverine) [5-7]. Hydrogen sulphate and methyl mercaptan constitute about $90 \%$ of the VSC present in breath. Hydrogen sulphate is mainly produced on the third posterior of the tongue. The methyl mercaptan and dimethyl sulphide are generated by the periodontal tissues. The condition necessary to produce these compounds is a basic environment. In fact, halitosis vanishes in the presence of sugar which activates the saccharolytic fermentation that, in turn, lowers the intraoral $\mathrm{pH}$, inhibiting the metabolic activity of the proteolytic microorganisms responsible for halitosis. Some new strategies, to prevent oral diseases, are developing to equilibrate oral microbiota by administrating probiotics [5]. Probiotics have been found to be beneficial to host health. According to World Health Organization (WHO) probiotics are "live microorganisms which, when administrated in adequate amounts, provide a health benefit to the host".

Their primary use in medicine has been for the management of intestinal tractproblems. On the other hand, recentstudies suggested a role of probiotics to promote oral health [8], in particular to reduce halitosis both in vitro and in vivo [6,8-19], but not all showed significant changes in the breath VSC concentration [20]. As one of the cause of halitosis coming from the disequilibrium of intestinal microbiota [4], in the present study the Probiotic Lactic Yeast $K$. marxianus B0399 was investigated to test its activity in reducing halitosis by acting on gastrointestinal microflora. K. marxianus B0399 is a strain belonging to a species naturally occurring in kefir and cheese [21]. It is a yeast closely related to Saccharomyces spp. [22], but it belongs to the genus Kluyveromyces, created by van der Walt [23]. K. marxianus B0399 was approved as a probiotic for both animal feeding [24] and human consumption [25], and its genomic DNA was completely sequenced [21]. It was demonstrated that $K$. marxianus B0399 remains viable after consumption surpassing the gastric barrier, adheres to the enterocytes of the intestinal epithelium, increases the concentration of good endogenous flora, in particular Bifidobacteria [26], and it regulates intestinal activity during antibiotic therapy, thanks to the yeast intrinsic resistance to bacterium-targeting antibiotics [27].

Furthermore, it has the capacity to produce enzymes such as $\beta$-galactosidase (reducing the effect of lactose intolerance in susceptible individuals) [28], $\beta$-glucosidase, inulinase, etc. It provides energy and promotes the growth of epithelial cells of colon by stimulating the production of short-chain fatty acids (SCFAs), such as acetate and propionate. It modulates the response of the immune system reducing the pro-inflammatory cytokine levels $[26,27,29]$, being useful for mitigation of the effect of several diseases, such as irritable bowel syndrome [29,30].

\section{Material and Methods}

A population of 115 patients of both sexes affected by halitosis, from ages 18 to 65, was considered. At the first visit, patients having cardiac problems, diabetic problems, immunosuppression, cancer, or already under conventional therapy for treating halitosis, and pregnant women were excluded. The selection of the group of patients was done at the end of rendering the sample as homogeneous as possible and excluding all the factors that could have falsified the result of the research. After the medical history interview, the patients were subjected to an instrumental survey of the breath to objectify the presence or lack of presence of halitosis quantifying it. The instrument used was a digital Halimeter connected to a computer that, through a specific software (Halisoft), monitored the levels of VSC present in the patient's breath. The patient's breath was tested three times within 10 minutes. For the first three minutes the patient breathed with mouth closed while the Halimeter tested the ambient air in order to have a comparison between environment VSC and the patient's expired VSC.

After three minutes, the patients put the disposable testing tube on the back of the tongue with open mouth and breathed for 30 seconds. During this period the Halimeter recorded the amount of VSC present in the breath of the patient. This cycle was repeated two more times. At the end of the data recording, the quantity of VSC on the graphic survey was evaluated. The threshold value was fixed at $100 \mathrm{ppb}$. If the recorded value was higher, the presence of halitosis was considered. The selected patients were treated with BioSympa ${ }^{\circledR}$ containing the Probiotic Lactic Yeast Kluyveromyces marxianus B0399, as active ingredient, and serum made from goat milk, enclosed in capsules. The dosage was one capsule, containing $10^{7} \mathrm{CFU}$ of $K$. marxianus B0399, three times a day before main meals. A package containing 50 pills of the product was given free of charge to the patients suffering from halitosis. The test performed with the digital halimeter was repeated at a distance of 7 to 14 days after the first survey and the first therapy treatment. In this period, no dental or other operations were performed.

\section{Discussion}

Bad breath is a common problem that can create social and relational difficulties. The role of probiotics in gastrointestinal tract is well-known, but in the last years they are investigated as an emerging resource in oral medicine. This concept prompts a new horizon on the relationship between diet and oral health. In fact, the present study shows that there is a correlation between intestinal microflora and halitosis. The administration of Biosympa ${ }^{\circledR}$ capsules, containing the probiotic lactic yeast $K$. marxianus B0399 at the concentration of $10^{7}$ ufc/cps, three times per day, showed to be effective in halitosis problems. $91 \%$ of the subjects, in fact, at the end of the treatment were completely recovered. It is well-known $[26,27,29]$ that $K$. marxianus B0399 increases the concentration of good endogenous intestinal flora and reduces the presence of pathogens and their associated VSC production. Based on our results, the administration of K. marxianus B0399 enclosed in capsules has been proven effective in halitosis by acting at the intestinal microbiome level. These data suggest the probiotic $K$. marxianus B0399 could be used to enhance oral benfits as well. 
Further clinical studies to provide more in-depth knowledge of $K$. marxianus B0399 in oral health will be needed. In fact, it cannot be ruled out that it would be effective directly in the oral cavity influencing and rebalancing the altered microflora responsible of unhealthy oral environment as well.

\section{Results}

115 patients were analysed. 35 of them were allowed to be included in the therapy because they showed VSC levels higher than $100 \mathrm{ppb}$, the fixed tresh-hold value. The average of the starting point value of VSC was 210.6 ppb (Figure 1). After 7 days the VSC values were reduced to $148.8 \mathrm{ppb}$. At the end of the treatment, after 14 days, in up to $91 \%$ of the cases, corresponding to 32 people, VSC values were lower than $100 \mathrm{ppb}$. Only 3 people showed VSC level still higher than $100 \mathrm{pb}$ at the end of the treatment. During and after the treatment with K. marxianus B0399 no collateral effects were reported from all patients.

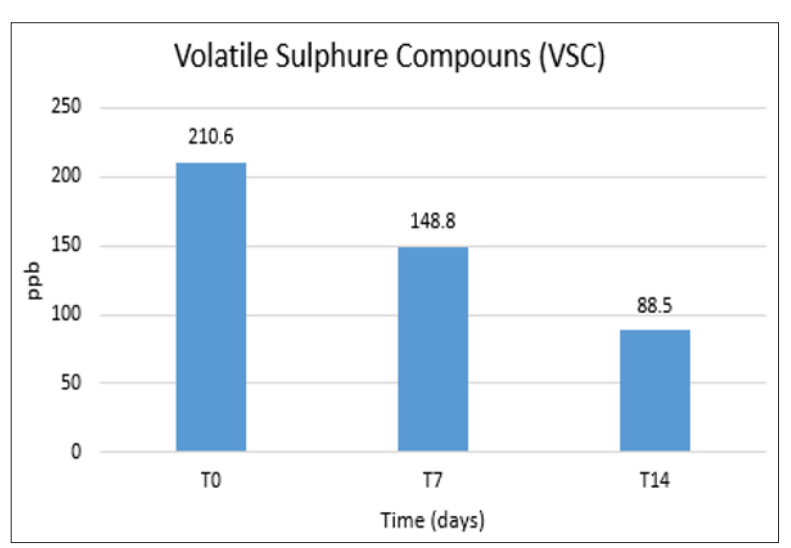

Figure 1: Volatile Sulphur Compounds (VSC) values. The graph shows VSC values recovered before starting the therapy (T0), and after 7 (T7) and 14 days (T14) of Kluyveromyces marxianus B0399 (Biosympa ${ }^{\circledR}$ capsules) administration. After 14 days of the treatment, $91 \%$ of the patients reached VSC values lower than $100 \mathrm{ppb}$.

\section{References}

1. Anusha RL, Umar D, Basheer B, Baroudi K (2015) The magic of magic bugs in oral cavity: Probiotics. J Adv Pharm Technol Res 6(2): 43-47.

2. Ruiz Martinez RC, Bedani R, Saad SM (2015) Scientific evidence for probiotics and prebiotics: An update for current prospectives and future challenges. Br J Nutr 114 (12): 1993-2015.

3. Miyazaki H, Arao M, Okamura K, Kawaguchi Y, Toyofuku A, et al. (1999) Tentative classification of halitosis and its treatment needs. Niigata Dental J 32: 7-11.

4. Henker J, Schuster F, Nissler K (2001) Successful treatment of gutcaused halitosis with a suspension of living non-pathogenic Escherichia coli bacteria - a case report. Eur J Pediatr 160: 592-594.

5. Mahasneh SA, Mahasneh AM (2017) Probiotics: A Promising Role in Dental Health. Dent J 5(4): 26.

6. Kang MS, Chung J, Kim SM, Yang KH, Oh JS (2006) Effect of Weissella cibaria isolates on the formation of Streptococcus mutans biofilm. Caries Res 40(5): 418-425.

7. Gupta G (2011) Probiotics and periodontal health. J Med Life 4 (4): 387-394.
8. Flichy Fernández AJ, Alegre Domingo T, Peñarrocha Oltra D, Peñarrocha Diago M (2010) Probiotic treatment in the oral cavity: An update. Med Oral Patol Oral Cir Bucal 15 (5): e677-e680.

9. Georgiou AC, Laine ML, Deng DM, Brandt BW, Van Loveren C, et al. (2018) Efficacy of probiotics: clinical and microbial parameters of halitosis. J Breath Res 12(4).

10. Masdea L, Kulik EM, Hauser Gerspach I, Ramseier AM, Filippi A, et al. (2012). Antimicrobial activity of Streptococcus salivarius K12 on bacteria involved in oral malodour. Arch Oral Bio 57(8): 1041-1047.

11. Iwamoto T, Suzuki N, Tanabe K, Takeshita T, Hirofuji T (2010) Effects of probiotic Lactobacillus salivarius WB21 on halitosis and oral health: An open-label pilot trial. Oral Surgery, Oral Med Oral Pathol Oral Radiol Endod 110(2): 201-208.

12. Suzuki N, Yoneda M, Tanabe K, Fujimoto A, Iha K, et al. (2014) Lactobacillus salivarius WB21-containing tablets for the treatment of oral malodor: a double-blind, randomized, placebo-controlled crossover trial. Oral Surg Oral Med Oral Pathol Oral Radiol 117(4): 462-470.

13. Jang HJ, Kang MS, Yi SH, Hong JY, Hong SP (2016) Comparative study on the characteristics of Weissella cibaria CMU and probiotic strains for oral care. Molecules 21(12): 1752.

14. Suzuki N, Higuchi T, Nakajima M, Fujimoto A, Morita H, et al. (2016) Inhibitory effect of Enterococcus faecium wb2000 on volatile sulfur compound production by porphyromonas gingivalis. Int J Dent 16: 1-5.

15. Lee SH, Baek DH (2014) Effects of Streptococcus thermophilus on volatile sulfur compounds produced by Porphyromonas gingivalis. Arch Oral Biol 59(11): 1205-1210.

16. Fujiwara N, Murakami K, Nakao M, Toguchi M, Yumoto H, et al. (2017) Novel reuterin-related compounds suppress odour by periodontopathic bacteria. Oral Dis 23(4): 492-497.

17. Keller MK, Bardow A, Jensdottir T, Lykkeaa J, Twetman S (2012) Effect of chewing gums containing the probiotic bacterium Lactobacillus reuteri on oral malodour. Acta Odontol Scan 70(3): 246-250.

18. Marchetti E, Tecco S, Santonico M, Vernile C, Ciciarelli D, et al. (2015) Multi-sensor approach for the monitoring of halitosis treatment via Lactobacillus brevis (CD2)-containing lozenges-a randomized, doubleblind placebo-controlled clinical trial. Sensors (Basel) 15(8): 1958319596.

19. Bosch M, Nart J, Audivert S, Bonachera MA, Alemany AS, et al. (2012) Isolation and characterization of probiotic strains for improving oral health. Arch Oral Biol 57(5): 539-549.

20. Allaker RP, Stephen AS (2017) Use of Probiotics and oral Health. Curr Oral Health Rep 4: 309-318.

21. Quarella S, Lovrovich P, Scalabrin S, Campedelli I, Backovic A, et al. (2016) Draft Genome Sequence of the Probiotic Yeast Kluyveromyces marxianus fragilis B0399. Genome Announc 4(5): e00923-e00916.

22. Lachance MA (2007) Current status on Kluyveromyces systematic. FEMS Yeast res 7: 642-645.

23. Van Der Walt JP (1956) Kluyveromyces - a new yeast genus of the Endomycetales. Antonie van Leeuwenhoek 22: 265-272.

24. (2004) EFSA, Opinion of the Scientific Panel on additives and products or substances used in animal feed (FEEDAP) on the safety of the microorganism product Turval B0399 for use as feed additive for weaned piglets. Question n. EFSA-Q-2003-051. EFSA J 28: 1-6.

25. (2016) Ministero della Salute. Registro degli integratori alimentari. Ministero della Salute, Rome, Italy.

26. Maccaferri S, Klinder A, Brigidi P, Cavina P, Costabile A (2012) Potential probiotic Kluyveromyces marxianus B0399 modulates the immune response in Caco-cells and peripheral blood mononuclear cells and impacts the human gut microbiota in an in vitro colonic model system. Appl Environ Microbiol 78: 956-964. 
27. Maccaferri S, Candela M, Turroni S, Centanni M, Severgnini M, et al. (2012) IBS-associated phylogenetic unbalances of the intestina microbiota are not reverted by probiotic supplementation. Gut Microbes 3: 406-413.

28. Fonseca GG, Heinzle E, Wittmann C, Gombert AK (2008) The yeast Kluyveromyces marxianus and its biotechnological potential. Appl Microbiol Biotechnol 79: 339-354.

ISSN: 2574-1241

DOI: $10.26717 /$ BJSTR.2018.12.002180

Cecchini F. Biomed J Sci \& Tech Res

(c) (P) This work is licensed under Creative

Submission Link: https://biomedres.us/submit-manuscript.php
29. Lisotti A, Cornia GL, Morselli Labate AM, Sartini A, Turco L, et al. (2013) Effects of a fermented milk containing Kluyveromyces marxianus B0399 and Bifidobacterium lactis BB12 in patients with irritable bowel syndrome. Minerva Gastroenterol Dietol 57: 1-12.

30. Nigro A, Nicastro A, Trodella R (2014) Retrospective observational study to investigate Sinerga, a multifactorial nutritional product, and bacterial extracts in the prevention of recurrent respiratory infections in children. Int J Immunopathol Pharmacol 27(3): 455-460.

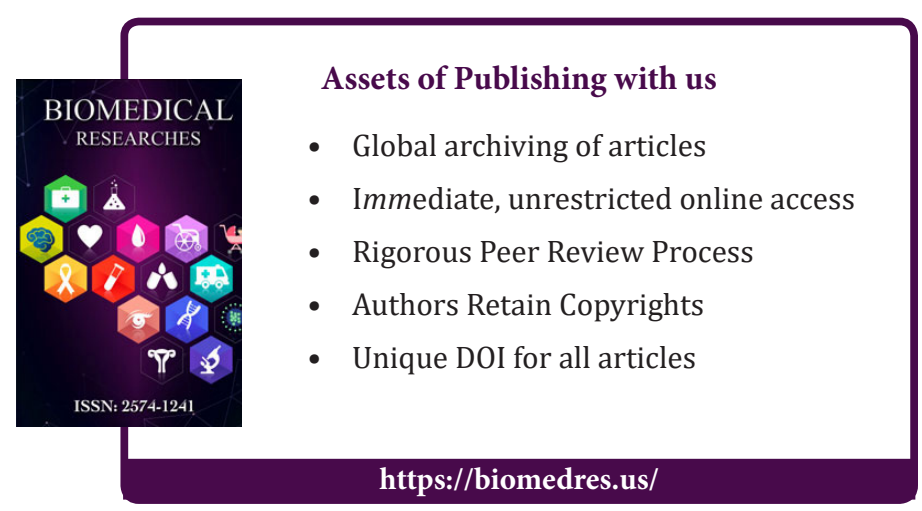

